\title{
Optimization of Access Threshold for Cognitive Radio Networks with Prioritized Secondary Users
}

\author{
Yuan Zhao, Jialiang Wang, Jiemin Liu, and Luyi Bai \\ School of Computer and Communication Engineering, Northeastern University at Qinhuangdao, Qinhuangdao 066004, China \\ Correspondence should be addressed to Yuan Zhao; yuanzh85@163.com
}

Received 29 January 2016; Accepted 1 June 2016

Academic Editor: Carlos Pomalaza-Ráez

Copyright (C) 2016 Yuan Zhao et al. This is an open access article distributed under the Creative Commons Attribution License, which permits unrestricted use, distribution, and reproduction in any medium, provided the original work is properly cited.

\begin{abstract}
We propose an access control scheme in cognitive radio networks with prioritized Secondary Users (SUs). Considering the different types of data in the networks, the SU packets in the system are divided into SU1 packets with higher priority and SU2 packets with lower priority. In order to control the access of the SU2 packets (including the new arrival SU2 packets and the interrupted SU2 packets), a dynamic access threshold is set. By building a discrete-time queueing model and constructing a three-dimensional Markov chain with the number of the three types of packets in the system, we derive some performance measures of the two types of the SU packets. Then, with numerical results, we show the change trends for the different performance measures. At last, considering the tradeoff between the throughput and the average delay of the SU2 packets, we build a net benefit function to make optimization for the access threshold.
\end{abstract}

\section{Introduction}

In conventional cognitive radio networks, two types of users, namely, Primary Users (PUs) and Secondary Users (SUs), share the spectrum resource in the system. The SUs can occupy the spectrum when the spectrum is not occupied by the PUs. The PUs have higher priority than the SUs and the PUs can interrupt the transmission of the SUs to take over the spectrum [1]. Most of existing researches for cognitive radio networks were studied under the assumption of single type of SUs [2-4]. However, in practical networks, there are different types of data in the system. So, it is necessary to consider the different types and prioritization among the SUs in cognitive radio networks.

In recent years, some researches began to focus on the performance analysis of the cognitive radio networks with prioritized SUs.

Lee et al. considered a cognitive radio network with three types of calls, namely, PU call, SU1 call, and SU2 call, in the system [5]. They denoted the high priority and the low priority SU calls as SU1 calls and SU2 calls. By building a continuous-time Markov chain and applying the Gauss-Seidel method, they gave the steady-state probability distribution of the system. Moreover, they presented some performance measures, such as the blocking rate and the throughput of the SU2 call.

Zhang et al. analyzed the transmission delay of prioritybased SUs in the opportunistic spectrum access (OSA) based cognitive radio networks [6]. By employing a preemptive resume priority (PRP) M/M/1 queuing model, they derived some performance measures, such as the transmission delay of the interrupted SUs with each priority level and the overall transmission delay of the interrupted SUs.

Zhao and Yue considered cognitive radio networks with multiple SUs [7]. A nonpreemptive priority scheme for the SU packets with higher priority was proposed and compared with the preemptive priority scheme. By constructing and analyzing a three-dimensional Markov chain, they derived the expression for the interrupted rate of the two types of SU packets, respectively.

However, the above researches about cognitive radio networks with prioritized SUs did not consider the access control for the SU packets with lower priority. In cognitive radio network, larger number of SU packets with lower priority which access the system without any restriction will disturb the transmission of the PUs and the SUs with higher priority. So, it is necessary to control the access of the SU packets 
with lower priority reasonably. In this paper, we consider setting an access threshold for the SU packets with lower priority (called SU2 packets) to control the access of the SU2 packets. Specially, we also make optimization for the access threshold, while the optimization research was not shown up in [5-7].

On the other hand, we can find that the researches about cognitive radio networks with priority SUs mentioned above were analyzed by using continuous-time models. However, considering the digital nature of model networks, the discrete-time models are more suitable when analyzing the system performance of the networks [8]. So, in this paper, we evaluate the system performance of the proposed cognitive radio networks with the discrete-time queueing analysis. By building a discrete-time queueing model with multiple priority levels, we construct a three-dimensional Markov chain and give the transition probability matrix of the Markov chain. Then with the obtained steady-state distribution, we derive some performance measures of the system. At last, we make optimization for the access threshold by building a net benefit function.

The remainder of this paper is organized as follows. The system model with model assumption and model analysis is demonstrated in Section 2. In Section 3, different performance measures for the SU1 packets and the SU2 packets are derived, respectively. In Section 4, numerical results for different performance measures are shown. Considering the tradeoff between different performance measures, the optimization for the access threshold is given in Section 5. Finally, conclusions are drawn in Section 6.

\section{System Model}

2.1. Model Assumption. We focus on a cognitive radio network with a single channel. The PU packets have higher priority to occupy the channel than the SU packets. There are two types of SU packets, namely, SU1 packets and SU2 packets. We assume that the SU1 packets have higher priority than the SU2 packets. That is to say, the PU packets have the highest priority, and the SU2 packets have the lowest priority. The PU packets can interrupt the transmission of the SU1 packets and the SU2 packets, while the SU1 packets can interrupt the transmission of the SU2 packets.

In order to reduce the latency of the PU packets and the SU1 packets, no buffers are set for the PU packets and the SU1 packets.

When a PU packet arrives at the system, if the channel is occupied by another PU packet, this newly arriving PU packet will leave the system to find another available channel directly. If the channel is occupied by an SU packet (an SU1 packet or an SU2 packet), this newly arriving PU packet will interrupt the transmission of the SU packet and occupy the channel.

When an SU1 packet arrives at the system, if the channel is occupied by a PU packet or an SU1 packet, this newly arriving SU1 packet will also leave the system to find another available channel. If the channel is occupied by an SU2 packet, this newly arriving SU1 packet will interrupt the transmission of the SU2 packet and occupy the channel.
A buffer called SU2 buffer is prepared for the SU2 packets. In order to control the access of the SU2 packets, an access threshold is set for the SU2 packets. When an SU2 packet arrives at the system, if the number of SU2 packets in the SU2 buffer is equal to the access threshold, this SU2 packet will be blocked by the system. The access threshold can also control the return action of the interrupted SU2 packet. When the transmission of an SU2 packet is interrupted, if the number of SU2 packets in the SU2 buffer is smaller than the access threshold, this interrupted SU2 packet can return back to the SU2 buffer; otherwise, this interrupted SU2 packet has to leave the system. Specially, we assume that the interrupted SU2 packets have higher priority than the newly arriving SU2 packets.

Based on the access control scheme mentioned above, we can build a queueing model with multiple priority levels and finite waiting room.

Considering the digital nature of modern networks, the time axis is assumed to be divided into slots with equal size. The slot boundary is denoted as $t=1,2, \ldots$. The arrival intervals of the PU packets, the SU1 packets, and the SU2 packets are assumed to follow geometric distribution with arrival rates $p_{1}, p_{21}$, and $p_{22}$. The transmission time of the PU packets, the SU1 packets, and the SU2 packets is assumed to follow geometric distribution with service rates $r_{1}, r_{21}$, and $r_{22}$. The access threshold is denoted as $T$, where $T>0$.

Let $L_{n}$ be the total number of packets (including PU packets, SU1 packets, and SU2 packets) in the system at the instant $t=n^{+}$. Let $S_{n}$ be the number of SU1 packets in the system at the instant $t=n^{+}$. Let $P_{n}$ be the number of PU packets in the system at the instant $t=n^{+}$. We note that $\left\{L_{n}, S_{n}, P_{n}\right\}$ constitutes a three-dimensional discrete-time Markov chain. With the access threshold $T$, we can give the state space $\Omega$ of $\left\{L_{n}, S_{n}, P_{n}\right\}$ as follows:

$$
\begin{aligned}
\Omega= & (0,0,0) \\
& \cup\{(i, 0,0) \cup(i, 1,0) \cup(i, 0,1): 1 \leq i \leq T+1\} .
\end{aligned}
$$

2.2. Model Analysis. Let $\mathbf{P}$ be the state transition probability matrix for the three-dimensional Markov chain $\left\{L_{n}, S_{n}, P_{n}\right\}$. $\mathbf{P}$ can be given as a $(T+2) \times(T+2)$ block-structured matrix as follows:

$$
\mathbf{P}=\left(\begin{array}{cccccc}
\mathbf{U}_{0} & \mathbf{V}_{0} & \mathbf{W}_{0} & & & \\
\mathbf{D}_{0} & \mathbf{C} & \mathbf{B} & \mathbf{A} & & \\
& \mathbf{D} & \mathbf{C} & \mathbf{B} & \mathbf{A} & \\
& \ddots & \ddots & \ddots & \ddots & \\
& & \text { D } & \text { C } & \text { B } & \text { A } \\
& & & \text { D } & \text { C } & \text { E } \\
& & & & \text { D } & \mathbf{F}
\end{array}\right) .
$$

Hereafter, we use the overbar notation to denote the probability of the complement of an event, for instance, $\bar{p}_{1}=1-p_{1}$. Moreover, we denote $\lambda=\bar{p}_{22} \bar{r}_{22}+p_{22} r_{22}, \xi=\bar{r}_{21}+p_{21} r_{21}$. The elements of $\mathbf{P}$ can be discussed by following subblock matrices. 
$\mathbf{U}_{0}$ is the probability for the total number of packets in the system being fixed at $0 . \mathbf{U}_{0}$ can be given as follows:

$$
\mathbf{U}_{0}=\bar{p}_{1} \bar{p}_{21} \bar{p}_{22} \text {. }
$$

$\mathbf{V}_{0}$ is the transition probability subblock when the total number of packets in the system transfers from 0 to $1 . \mathbf{V}_{0}$ can be given as follows:

$$
\mathbf{V}_{0}=\left(\bar{p}_{1} \bar{p}_{21} p_{22}, \bar{p}_{1} p_{21} \bar{p}_{22}, p_{1} \bar{p}_{22}\right) .
$$

$\mathbf{W}_{0}$ is the transition probability subblock when the total number of packets in the system transfers from 0 to 2 . $\mathbf{W}_{0}$ can be given as follows:

$$
\mathbf{W}_{0}=\left(0, \bar{p}_{1} p_{21} p_{22}, p_{1} p_{22}\right) .
$$

$\mathbf{D}_{0}$ is the transition probability subblock when the total number of packets in the system transfers from 1 to $0 . \mathbf{D}_{0}$ can be given as follows:

$$
\mathbf{D}_{0}=\left(\bar{p}_{1} \bar{p}_{21} \bar{p}_{22} r_{22}, \bar{p}_{1} \bar{p}_{21} \bar{p}_{22} r_{21}, \bar{p}_{1} \bar{p}_{21} \bar{p}_{22} r_{1}\right)^{\mathrm{T}},
$$

where $\mathrm{T}$ describes the transpose operator of the matrix.

D is the transition probability subblock when the total number of packets in the system transfers from $u$ to $(u-1)$, where $2 \leq u \leq T+1$. $\mathrm{D}$ can be given as follows:

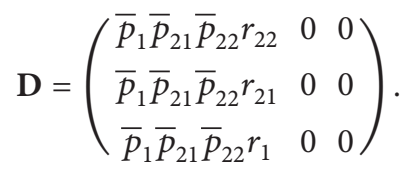

C is the transition probability subblock when the total number of packets in the system transfers from $u$ to $u$, where $1 \leq u \leq T$. C can be given as follows:

$$
\mathbf{C}=\left(\begin{array}{ccc}
\bar{p}_{1} \bar{p}_{21} \lambda & \bar{p}_{1} p_{21} \bar{p}_{22} r_{22} & p_{1} \bar{p}_{22} r_{22} \\
\bar{p}_{1} \bar{p}_{21} p_{22} r_{21} & \bar{p}_{1} \bar{p}_{22} \xi & \bar{p}_{22} p_{1} \\
\bar{p}_{1} \bar{p}_{21} p_{22} r_{1} & \bar{p}_{1} p_{21} \bar{p}_{22} r_{1} & \bar{p}_{22}\left(\bar{r}_{1}+r_{1} p_{1}\right)
\end{array}\right) \text {. }
$$

B is the transition probability subblock when the total number of packets in the system transfers from $u$ to $(u+1)$, where $1 \leq u \leq T-1$. B can be given as follows:

$$
\mathbf{B}=\left(\begin{array}{ccc}
\bar{p}_{1} \bar{p}_{21} p_{22} \bar{r}_{22} & \bar{p}_{1} p_{21} \lambda & p_{1} \lambda \\
0 & \bar{p}_{1} p_{22} \xi & p_{22} p_{1} \\
0 & \bar{p}_{1} p_{21} p_{22} r_{1} & p_{22}\left(\bar{r}_{1}+r_{1} p_{1}\right)
\end{array}\right) .
$$

A is the transition probability subblock when the total number of packets in the system transfers from $u$ to $(u+2)$, where $1 \leq u \leq T-1$. A can be given as follows:

$$
\mathbf{A}=\left(\begin{array}{ccc}
0 & \bar{p}_{1} p_{21} p_{22} \bar{r}_{22} & p_{1} p_{22} \bar{r}_{22} \\
0 & 0 & 0 \\
0 & 0 & 0
\end{array}\right)
$$

$\mathbf{E}$ is the transition probability subblock when the total number of packets in the system transfers from $T$ to $(T+1)$. $\mathbf{E}$ can be given as follows:

E

$$
=\left(\begin{array}{ccc}
\bar{p}_{1} \bar{p}_{21} p_{22} r_{22} & \bar{p}_{1} p_{21}\left(1-\bar{p}_{22} r_{22}\right) & p_{1}\left(1-\bar{p}_{22} r_{22}\right) \\
0 & \bar{p}_{1} p_{22} \xi & p_{22} p_{1} \\
0 & \bar{p}_{1} p_{21} p_{22} r_{1} & p_{22}\left(\bar{r}_{1}+r_{1} p_{1}\right)
\end{array}\right) .
$$

$\mathbf{F}$ is the transition probability subblock when the total number of packets in the system is fixed at $(T+1)$. $\mathbf{F}$ can be given as follows:

$$
\mathbf{F}=\left(\begin{array}{ccc}
\bar{p}_{1} \bar{p}_{21}\left(1-\bar{p}_{22} r_{22}\right) & \bar{p}_{1} p_{21} & p_{1} \\
\bar{p}_{1} \bar{p}_{21} p_{22} r_{21} & \bar{p}_{1} \xi & p_{1} \\
\bar{p}_{1} \bar{p}_{21} p_{22} r_{1} & \bar{p}_{1} p_{21} r_{1} & \bar{r}_{1}+r_{1} p_{1}
\end{array}\right) .
$$

The structure of the transition probability matrix $\mathbf{P}$ indicates that the three-dimensional Markov chain $\left\{L_{n}, S_{n}, P_{n}\right\}$ is nonperiodic, irreducible, and positive recurrent [9]. The steady-state distribution $\pi_{i, j, k}$ of the three-dimensional Markov chain is defined as follows:

$$
\pi_{i, j, k}=\lim _{n \rightarrow \infty} P\left\{L_{n}=i, S_{n}=j, P_{n}=k\right\} .
$$

Let $\Pi$ be the steady-state probability vector, which is the unique solution of equations $\Pi \mathbf{P}=\Pi$, $\Pi \mathbf{e}=1$, where $\mathbf{e}$ is a one column vector.

We partition $\Pi$ as $\Pi=\left(\Pi_{0}, \Pi_{1}, \ldots, \Pi_{T}, \Pi_{T+1}\right)$, where $\Pi_{0}=\pi_{0,0,0}$ and $\Pi_{i}=\left(\pi_{i, 0,0}, \pi_{i, 1,0}, \pi_{i, 0,1}\right)$ for $1 \leq i \leq T+1$. By applying a Gauss-Seidel method, we can obtain the steadystate probability vector $\Pi$.

\section{Performance Measures}

3.1. Performance Measures of the SU1 Packets. The average queue length $E[S U 1]$ of the SU1 packets is defined as the number of SU1 packets in the system per slot. We can give the expression of the average queue length $E[S U 1]$ of the SU1 packets as follows:

$$
E[\mathrm{SU} 1]=\sum_{i=1}^{T+1} \pi_{i, 1,0} .
$$

The blocking rate $\beta_{21}$ of the SU1 packets is defined as the number of SU1 packets that are blocked by the system per slot. We can give the expression of the blocking rate $\beta_{21}$ of the SU1 packets as follows:

$$
\begin{aligned}
\beta_{21} & \\
& =p_{21}\left(\sum_{i=1}^{T+1}\left(\pi_{i, 1,0}\left(\bar{r}_{21}+r_{21} p_{1}\right)+\pi_{i, 0,1}\left(\bar{r}_{1}+r_{1} p_{1}\right)\right)\right. \\
& \left.+\sum_{i=0}^{T+1} \pi_{i, 0,0} p_{1}\right) .
\end{aligned}
$$


The interrupted rate $\gamma_{21}$ of the SU1 packets is defined as the number of SU1 packets that are interrupted by the PU packets per slot. We can give the expression of the interrupted rate $\gamma_{21}$ of the SU1 packets as follows:

$$
\gamma_{21}=\sum_{i=1}^{T+1} \pi_{i, 1,0} \bar{r}_{21} p_{1} .
$$

The throughput $\theta_{21}$ of the SU1 packets is defined as the number of SU1 packets that are transmitted completely by the system per slot. We can give the expression of the throughput $\theta_{21}$ of the SU1 packets as follows:

$$
\theta_{21}=p_{21}-\beta_{21}-\gamma_{21} \text {. }
$$

3.2. Performance Measures of the SU2 Packets. The average queue length $E[S U 2]$ of the SU2 packets is defined as the number of SU2 packets in the system per slot. We can give the expression of the average queue length $E$ [SU2] of the SU2 packets as follows:

$$
E[\mathrm{SU} 2]=\sum_{i=0}^{T+1} i \pi_{i, 0,0}+\sum_{i=1}^{T+1}(i-1)\left(\pi_{i, 1,0}+\pi_{i, 0,1}\right) .
$$

The blocking rate $\beta_{22}$ of the SU2 packets is defined as the number of SU2 packets that are blocked by the system per slot. We can give the expression of the blocking rate $\beta_{22}$ of the SU2 packets as follows:

$$
\begin{aligned}
\beta_{22} & =p_{22}\left(\pi_{T+1,0,0}\left(1-r_{22} \bar{p}_{1} \bar{p}_{21}\right)\right. \\
& \left.+\pi_{T+1,1,0}\left(1-r_{21} \bar{p}_{1} \bar{p}_{21}\right)\right) \\
& +p_{22}\left(\pi_{T+1,0,1}\left(1-r_{1} \bar{p}_{1} \bar{p}_{21}\right)\right. \\
& \left.+\pi_{T, 0,0} \bar{r}_{22}\left(1-\bar{p}_{1} \bar{p}_{21}\right)\right) .
\end{aligned}
$$

The interrupted losing rate $\gamma_{22}$ of the SU2 packets is defined as the number of SU2 packets that are interrupted by the PU packets or the SU1 packets and being forced to leave the system because of the number of SU2 packets in the buffer achieving the access threshold. We can give the expression of the interrupted losing rate $\gamma_{22}$ of the SU2 packets as follows:

$$
\gamma_{22}=\pi_{T+1,0,0} \bar{r}_{22}\left(1-\bar{p}_{1} \bar{p}_{21}\right) .
$$

The throughput $\theta_{22}$ of the SU2 packets is defined as the number of SU2 packets that are transmitted completely by the system per slot. We can give the expression of the throughput $\theta_{22}$ of the SU2 packets as follows:

$$
\theta_{22}=p_{22}-\beta_{22}-\gamma_{22} \text {. }
$$

The average delay $\delta_{22}$ of the SU2 packets is defined as the average time length from an SU2 packet joining the system to this SU2 packet leaving the system (being transmitted completely or being interrupted to leave). With Little's formula [10], we can give the expression of the average delay $\delta_{22}$ of the SU2 packets as follows:

$$
\delta_{22}=\frac{E[\mathrm{SU} 2]}{p_{22}-\beta_{22}} .
$$

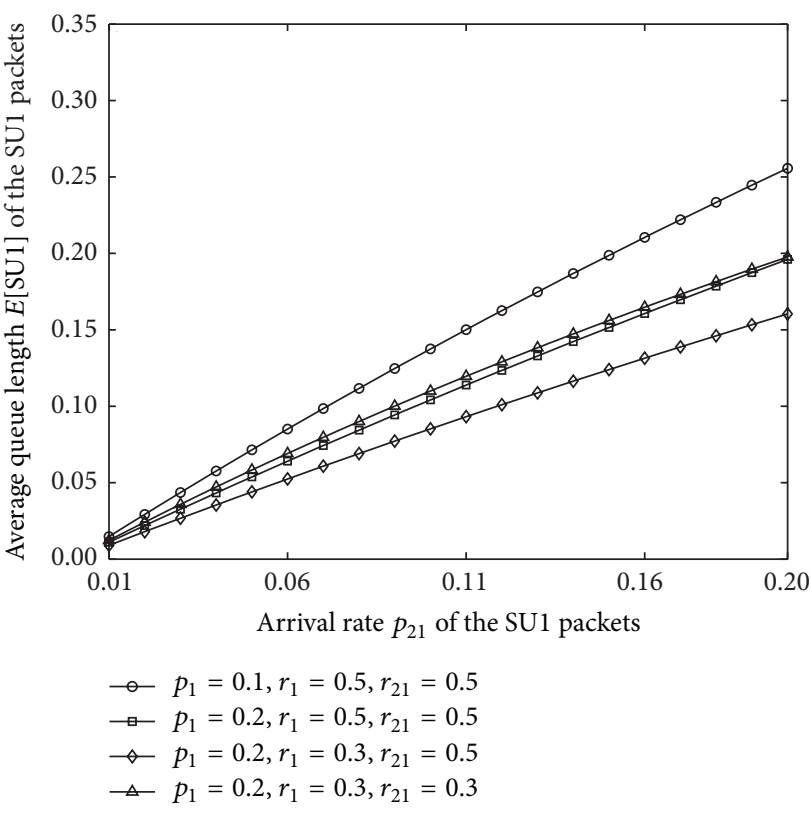

FIGURE 1: Average queue length $E[S U 1]$ of the SU1 packets.

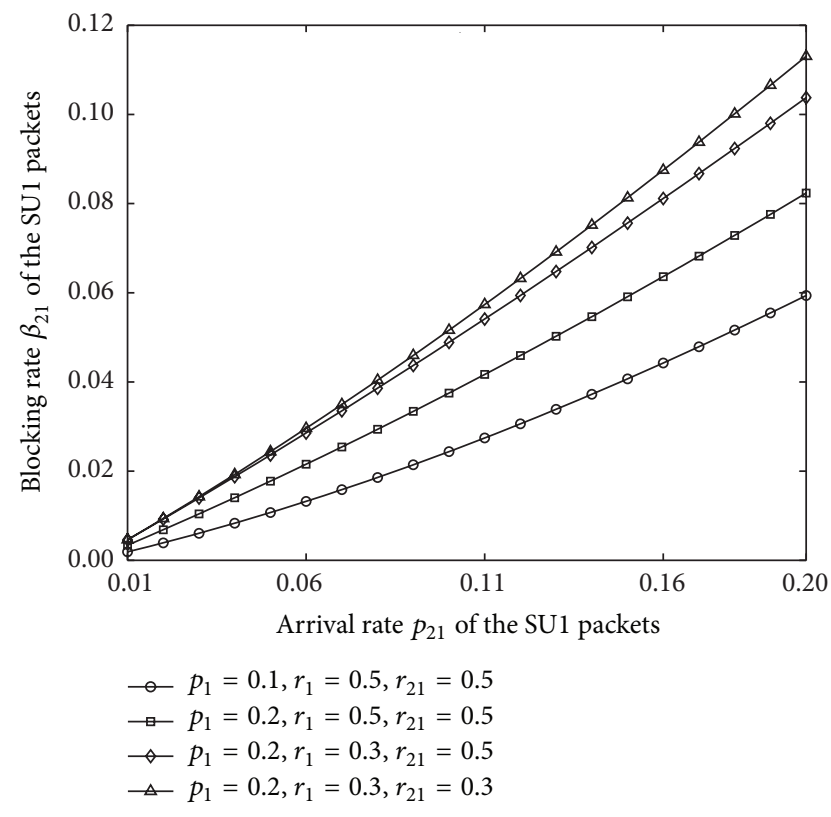

FIGURE 2: Blocking rate $\beta_{21}$ of the SU1 packets.

\section{Numerical Results}

In this section, we show the change trends for different performance measures with numerical results for the SU1 packets and the SU2 packets, respectively.

4.1. Numerical Results for the SU1 Packets. According to the working principle of the system model, the performance of the SU1 packets will be influenced by the PU packets. Figures 1-3 show the change trends for the average queue length $E[\mathrm{SU} 1]$, the blocking rate $\beta_{21}$, and the throughput $\theta_{21}$ of the 


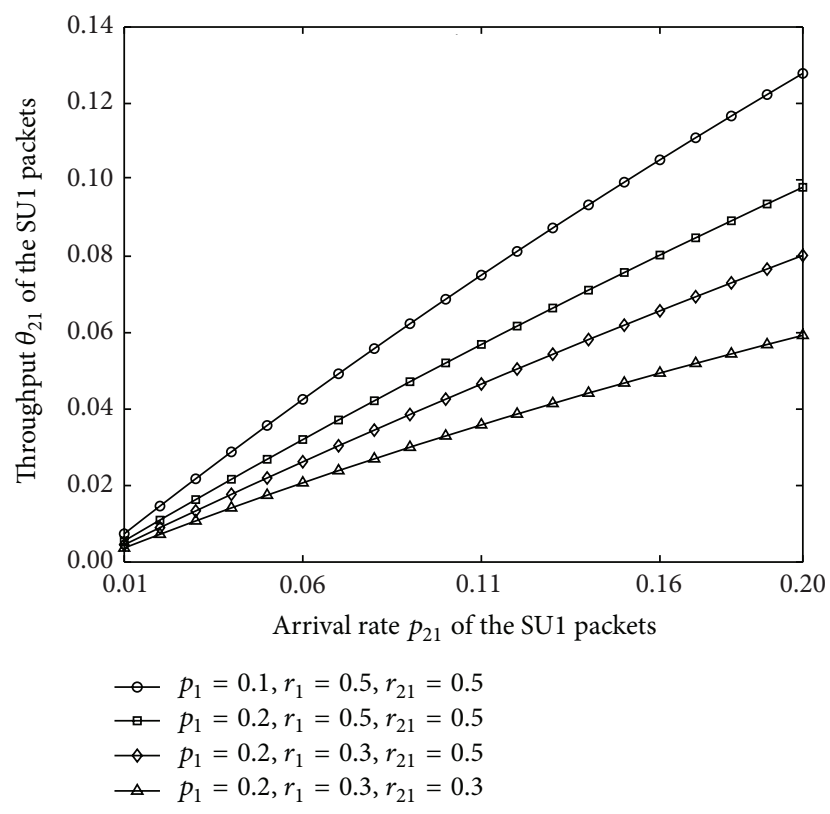

FIgURE 3: Throughput $\theta_{21}$ of the SU1 packets.

SU1 packets with different parameter settings of PU packets and SU1 packets.

From Figures 1-3, we find that as the arrival rate $p_{21}$ of the SU1 packets increases, the average queue length $E$ [SU1], the blocking rate $\beta_{21}$, and the throughput $\theta_{21}$ of the SU1 packets will increase. This is because the larger the arrival rate of the SU1 packets is, the more the SU1 packets will join the system. As a result, the average queue length of the SU1 packets in the system will be higher. On the other hand, the larger the number of SU1 packets joining the system is, the more the possibility for the SU1 packets being blocked by the system is and the higher the blocking rate of the SU1 packets will be. Moreover, the more the SU1 packets joining the system are, the more the SU1 packets will be transmitted and the greater the throughput of the SU1 packets will be.

From Figures 1-3, we also find that as the arrival rate $p_{1}$ of the PU packets increases, the blocking rate $\beta_{21}$ of the SU1 packets will increase and the average queue length $E[S U 1]$ and the throughput $\theta_{21}$ of the SU1 packets will decrease. The reason is that as the arrival rate of the PU packets increases, the possibility for the channel being occupied by the PU packets will also increase. As a result, the number of SU1 packets joining the system and being transmitted completely will decrease, and this will decrease the average queue length and the throughput of the SU1 packets. Moreover, larger number of SU1 packets will be blocked by the system, and the blocking rate of the SU1 packets will be increased.

Moreover, from Figures 1-3, we conclude that as the service rate $r_{1}$ of the PU packets increases, the average queue length $E[\mathrm{SU} 1]$ and the throughput $\theta_{21}$ of the SU1 packets will increase and the blocking rate $\beta_{21}$ of the SU1 packets will decrease. This is because the higher the service rate of the PU packets is, the larger the possibility for the SU1 packets occupying the channel is, and this will increase the average

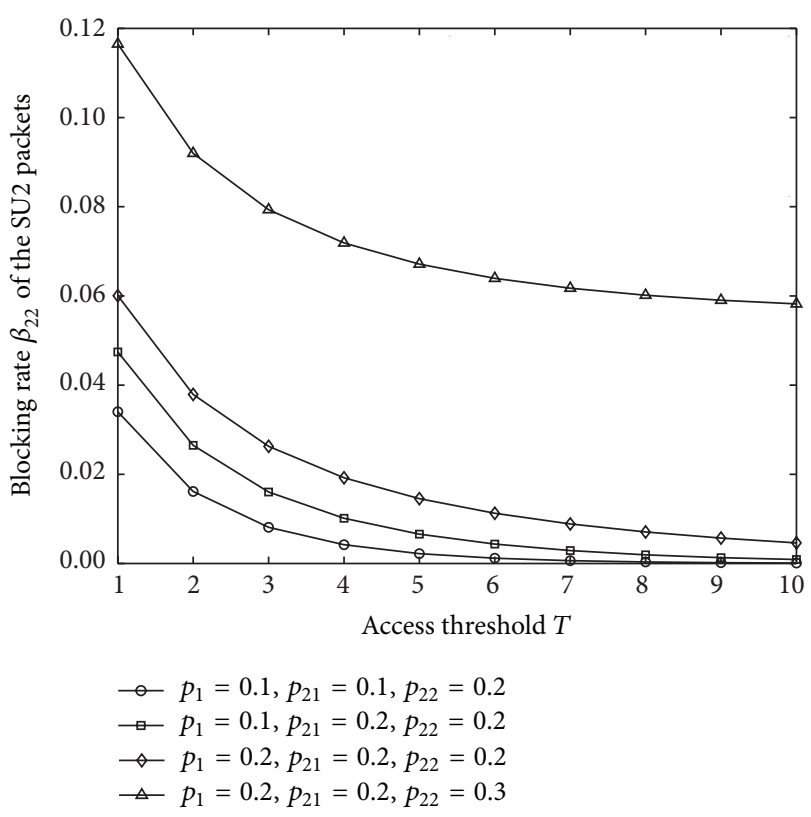

FIGURE 4: Blocking rate $\beta_{22}$ of the SU2 packets.

queue length and the throughput of the SU1 packets. At the same time, the blocking rate of the SU1 packets will be lower.

Additionally, Figures 1-3 show that as the service rate $r_{21}$ of the SU1 packets increases, the average queue length $E$ [SU1] and the blocking rate $\beta_{21}$ of the SU1 packets will decrease and the throughput $\theta_{21}$ of the SU1 packets will increase. The reason is that as the service rate of the SU1 packets increases, the SU1 packets on the channel will be transmitted more quickly and then the throughput of the SU1 packets will be greater. Along with the increase of the service rate of the SU1 packets, the average number of SU1 packets in the system will be lower and then the average queue length and the blocking rate of the SU1 packets will decrease.

4.2. Numerical Results for the SU2 Packets. According to the working principle of the system model, the performance of the SU2 packets will be influenced not only by the PU packets but also by the SU1 packets. Figures 4-6 show the change trends for the blocking rate $\beta_{22}$, the throughput $\theta_{22}$, and the average delay $\delta_{22}$ of the SU2 packets with respect to the access threshold under different parameter settings of PU packets and SU1 packets. In Figures 4-6, without loss of generality, the service rates $r_{1}, r_{21}$, and $r_{22}$ are assumed to be fix at 0.5 .

From Figures $4-6$, we find that as the access threshold $T$ increases, the blocking rate $\beta_{22}$ of the SU2 packets will decrease and the throughput $\theta_{22}$ and the average delay $\delta_{22}$ of the SU2 packets will increase. This is because the higher the access threshold is, the more the SU2 packets can be admitted to join the system and the more the SU2 packets will wait in the system. As a result, the average delay of the SU2 packets will be higher. On the other hand, as the access threshold increases, the number of SU2 packets being blocked by system will decrease and then the blocking rate of the SU2 packets will decrease too. Moreover, the more the SU2 packets joining the system are, the more the SU2 packets will be 


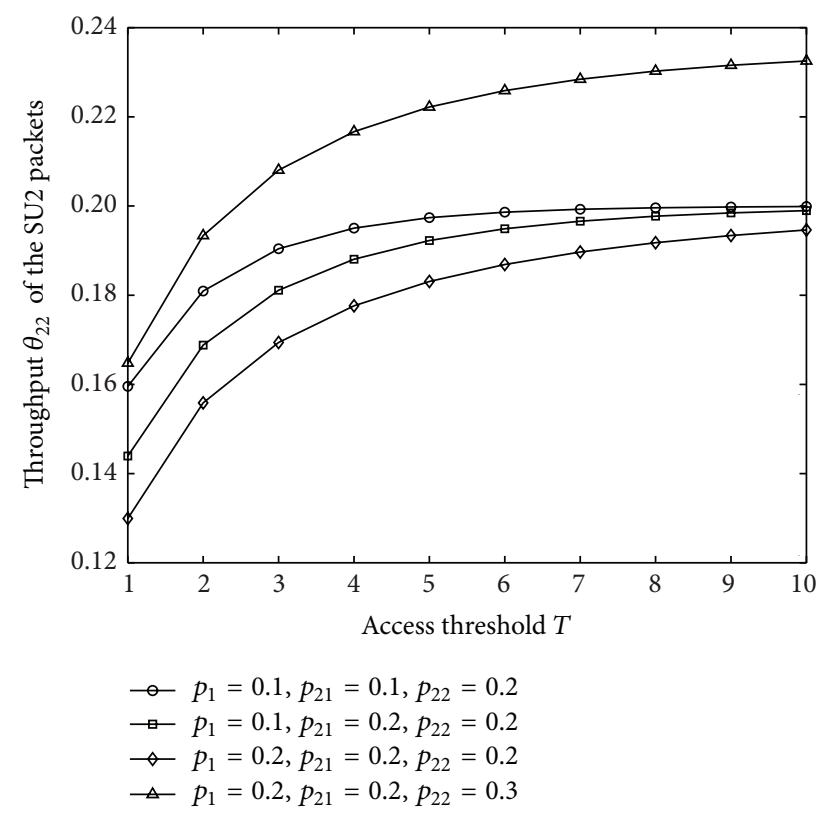

FIgURE 5: Throughput $\theta_{22}$ of the SU2 packets.

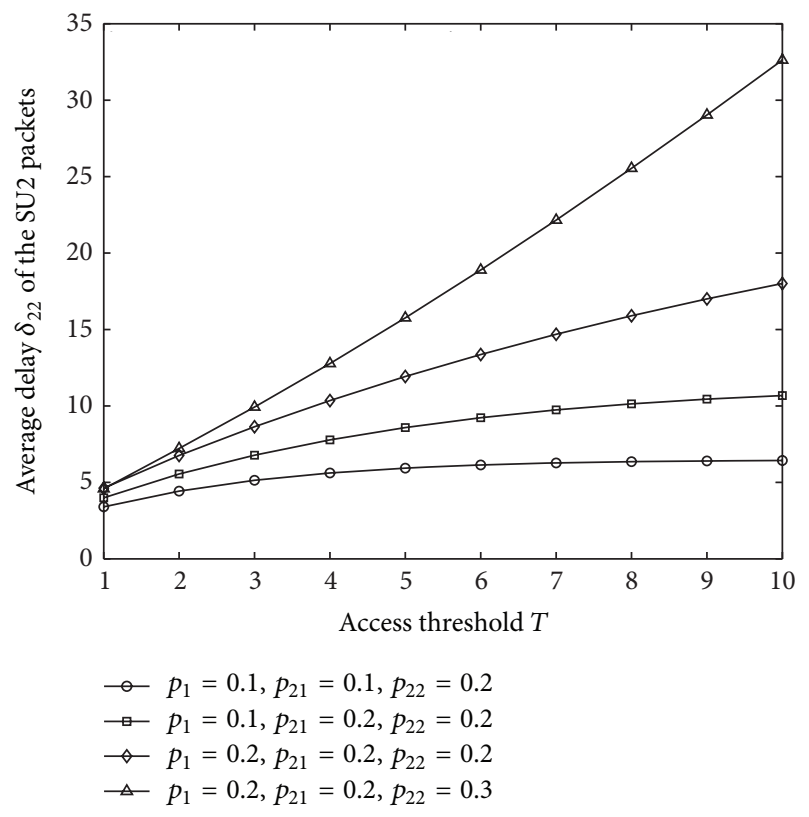

FIGURE 6: Average delay $\delta_{22}$ of the SU2 packets.

transmitted completely and the greater the throughput of the SU2 packets will be. Specially, from Figure 4, we find that as the access threshold $T$ increases, the blocking rete $\beta_{22}$ of the SU2 packets will decrease. As a result, the number of SU2 packets being in the system will increase and the possibility for the channel being occupied will be higher. Therefore, we conclude that the possibility for the channel being occupied can be increased by setting a greater access threshold.

From Figures 4-6, we also find that as the arrival rate $p_{1}$ of the PU packets or the arrival rate $p_{21}$ of the SU1 packets increases, the blocking rate $\beta_{22}$ and the average delay $\delta_{22}$ of the SU2 packets will increase and the throughput $\theta_{22}$ of the SU2 packets will decrease. The reason is that as the arrival rate of the PU packets or the arrival rate of the SU1 packets increases, the possibility for the channel being occupied by the SU2 packets will decrease. Then the number of SU2 packets waiting in the system will increase, and this will induce the increase in the average delay and the blocking rate of the SU2 packets. Moreover, the less the number of SU2 packets occupying the channel is, the less the number of SU2 packets will be transmitted completely. As a result, the throughput of the SU2 packets will be lower.

Additionally, Figures $4-6$ show that as the arrival rate $p_{22}$ of the SU2 packets increases, the blocking rate $\beta_{22}$, the throughput $\theta_{22}$, and the average delay $\delta_{22}$ of the SU2 packets will increase too. The reason is that as the arrival rate of the SU2 packets increases, the number of SU2 packets with joining need will increase. The more the SU2 packets joining the system are, the more the SU2 packets waiting in the system are and the more the SU2 packets will be transmitted completely. So the blocking rate, the throughput, and the average delay of the SU2 packets will be higher.

\section{Optimization for the Access Threshold}

In this section, we focus on the optimal setting for the access threshold of the SU2 packets. From the numerical results, we conclude that as the access threshold $T$ increases, the throughput $\theta_{22}$ of the SU2 packets increases, and this is what we want to see. On the other hand, as the access threshold $T$ increases, the average delay $\delta_{22}$ of the SU2 packets also increases, which is not what we want to see. So in order to balance the throughput of the SU2 packets and the average delay of the SU2 packets, we build a net benefit function $F(T)$ with the access threshold $T$ as follows:

$$
F(T)=b \theta_{22}-c \delta_{22},
$$

where $b$ and $c$ are the impact factors for the net benefit function. Moreover, $b$ and $c$ can be set according to the actual network situation.

From (23), we can derive the optimal access threshold $T^{*}$ as follows:

$$
T^{*}=\underset{T}{\arg \max }\{F(T)\} .
$$

In order to find the optimal access threshold $T^{*}$, by setting $b=300$ and $c=2$ as an example, we depict the change trend for the net benefit function $F(T)$ in Figure 7.

From Figure 7, we find that as the access threshold increases, the net benefit function shows an upper convex change trend. The reason may be that when the access threshold is smaller, the access threshold is the main factor to control the access actions of the SU2 packets. As the access threshold increases, the throughput will increase quickly, which will induce the increasing trend of the net benefit function. However, as the access threshold continues to increase, the average delay of the SU2 packets increases rapidly, which will cause the decrease of the net benefit. So, from Figure 7, we can find that there exists an optimal access threshold to 


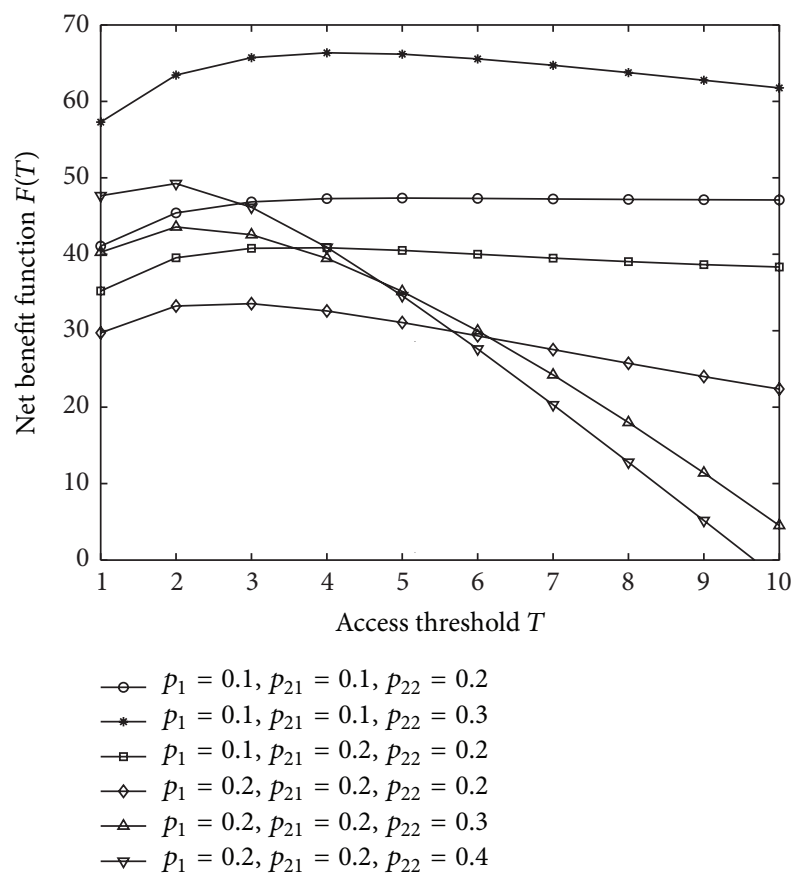

FIGURE 7: Change trend for the net benefit function $F(T)$.

TABLE 1: Numerical results for the optimal access threshold and the corresponding net benefit.

\begin{tabular}{lcccc}
\hline$p_{1}$ & $p_{21}$ & $p_{22}$ & $T^{*}$ & $F\left(T^{*}\right)$ \\
\hline 0.1 & 0.1 & 0.2 & 5 & 47.3504 \\
0.1 & 0.1 & 0.3 & 4 & 66.3590 \\
0.1 & 0.2 & 0.2 & 4 & 40.8567 \\
0.2 & 0.2 & 0.2 & 3 & 33.5409 \\
0.2 & 0.2 & 0.3 & 2 & 43.5728 \\
0.2 & 0.2 & 0.4 & 2 & 49.2551 \\
\hline
\end{tabular}

achieve the maximum net benefit. We summarize the optimal access threshold and the corresponding net benefit in Table 1 for different parameter settings.

From Table 1, we conclude that as the arrival rate $p_{1}$ of the PU packets or the arrival rate $p_{21}$ of the SU1 packets increases, the optimal access threshold $T^{*}$ shows a decreasing tendency. This is because as the arrival rate of the PU packets increases or the arrival rate $p_{21}$ of the SU1 packets increases, the possibility for the SU2 packets occupying the channel will be lower and larger number of SU2 packets have to wait in the system, which will result in the increase of the average delay of the SU2 packets. In order to reduce the average delay of the SU2 packets, the access threshold should be set lower.

On the other hand, from Table 1, we find that as the arrival rate $p_{22}$ of the SU2 packets increases, the optimal access threshold $T^{*}$ shows a decreasing tendency when we compare $p_{1}=0.1, p_{21}=0.1, p_{22}=0.2$ with $p_{1}=0.1, p_{21}=0.1, p_{22}=$ 0.3 and $p_{1}=0.2, p_{21}=0.2, p_{22}=0.2$ with $p_{1}=0.2, p_{21}=0.2$, $p_{22}=0.3$. The reason for the interesting tendency may be that the higher the arrival rate of the SU2 packets is, the more the SU2 packets will join the system, which will induce an increase in the average delay of the SU2 packets. So, in order to reduce the average delay of the SU2 packets, the access threshold should be set lower. Moreover, from Table 1, we also find that for $p_{1}=0.2, p_{21}=0.2$, the optimal access threshold $T^{*}$ will be fixed at $T^{*}=2$ when the arrival rate of the SU2 packets increases from $p_{22}=0.3$ to $p_{22}=0.4$. The reason for this change trend may be that when the access threshold is decreased to a low level, considering the tradeoff between the average delay and the throughput of the SU2 packets, the optimal access threshold will not be decreased in order to guarantee the throughput of the SU2 packets.

Therefore, from the numerical results shown in Table 1, we conclude that we should set the access threshold based on the different network running status in practice.

\section{Conclusion}

In this paper, we consider a cognitive radio network with prioritized SU packets. In order to control the access of the SU2 packets (i.e., the SU packets with lower priority), an access threshold was set not only for the newly arriving SU2 packets but also for the interrupted SU2 packets. By building and analyzing a discrete-time Markov chain, some performance measures for the two kinds of SU packets (SU1 packets and SU2 packets) were derived. With numerical results, we investigated the change trends for different performance measures and found that there was a tradeoff between the throughput and the average delay of the SU2 packets. At last, by constructing a net benefit function, we derived the numerical results for the optimal access threshold which balanced different performance measures of the SU2 packets.

\section{Competing Interests}

The authors declare that the funding mentioned in Acknowledgments section does not lead to any conflict of interests. Additionally, the authors declare that there is no conflict of interests regarding the publication of this paper.

\section{Acknowledgments}

This work was supported by the Doctoral Fund Project of Northeastern University at Qinhuangdao (XNB201606), the National Natural Science Foundation of China (61402087), the Natural Science Foundation of Hebei Province (F2016501073 and F2015501049), the Scientific Research Fund of Hebei Education Department (QN2016307), and the Fundamental Research Fund for the Central Universities (N152303007), China.

\section{References}

[1] E. Z. Tragos, S. Zeadally, A. G. Fragkiadakis, and V. A. Siris, "Spectrum assignment in cognitive radio networks: a comprehensive survey," IEEE Communications Surveys \& Tutorials, vol. 15, no. 3, pp. 1108-1135, 2013.

[2] S. Tang, "A general model of opportunistic spectrum sharing with unreliable sensing," International Journal of Communication Systems, vol. 27, no. 1, pp. 31-44, 2014. 
[3] S. Jin, Y. Zhao, W. Yue, and Z. Saffer, "Performance analysis and optimization of an adaptive admission control scheme in cognitive radio networks," Mathematical Problems in Engineering, vol. 2013, Article ID 727310, 2013.

[4] J. Wang, A. Huang, W. Wang, and T. Q. S. Quek, “Admission control in cognitive radio networks with finite queue and user impatience," IEEE Wireless Communications Letters, vol. 2, no. 2, pp. 175-178, 2013.

[5] Y. Lee, C. G. Park, and D. B. Sim, "Cognitive radio spectrum access with prioritized secondary users," Applied Mathematics \& Information Sciences, vol. 6, no. 2, supplement, pp. 595S-601S, 2012.

[6] Y. Zhang, T. Jiang, L. Zhang, D. Qu, and W. Peng, "Analysis on the transmission delay of priority-based secondary users in cognitive radio networks," in Proceedings of the International Conference on Wireless Communications and Signal Processing (WCSP '13), IEEE Press, Hangzhou, China, October 2013.

[7] Y. Zhao and W. Yue, "Performance comparison between two kinds of priority schemes in cognitive radio networks," in Queueing Theory and Network Applications, T. V. Do, Y. Takahashi, W. Yue, and V.-H. Nguyen, Eds., vol. 383 of Advances in Intelligent Systems and Computing, pp. 73-80, Springer, New York, NY, USA, 2016.

[8] H. Takagi, Queueing Analysis, Volume 3: Discrete-Time Systems, North-Holland Elsevier, Amsterdam, The Netherlands, 1993.

[9] A. S. Alfa, Queueing Theory for Telecommunication: Discrete Time Modelling of a Single Node System, Springer, New York, NY, USA, 2010

[10] N. Tian and Z. G. Zhang, Vacation Queueing Models: Theory and Applications, Springer, New York, NY, USA, 2006. 

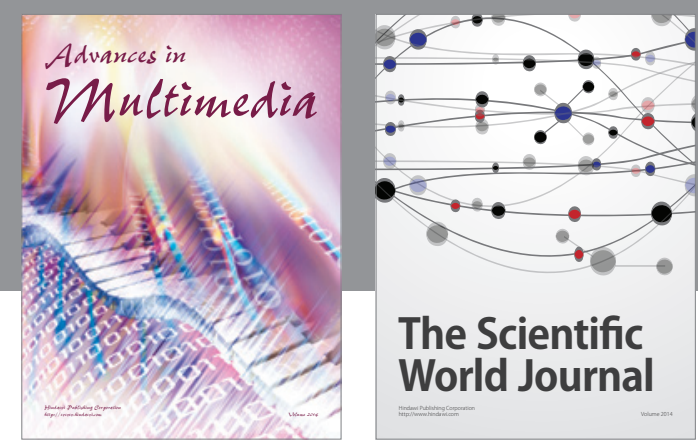

The Scientific World Journal
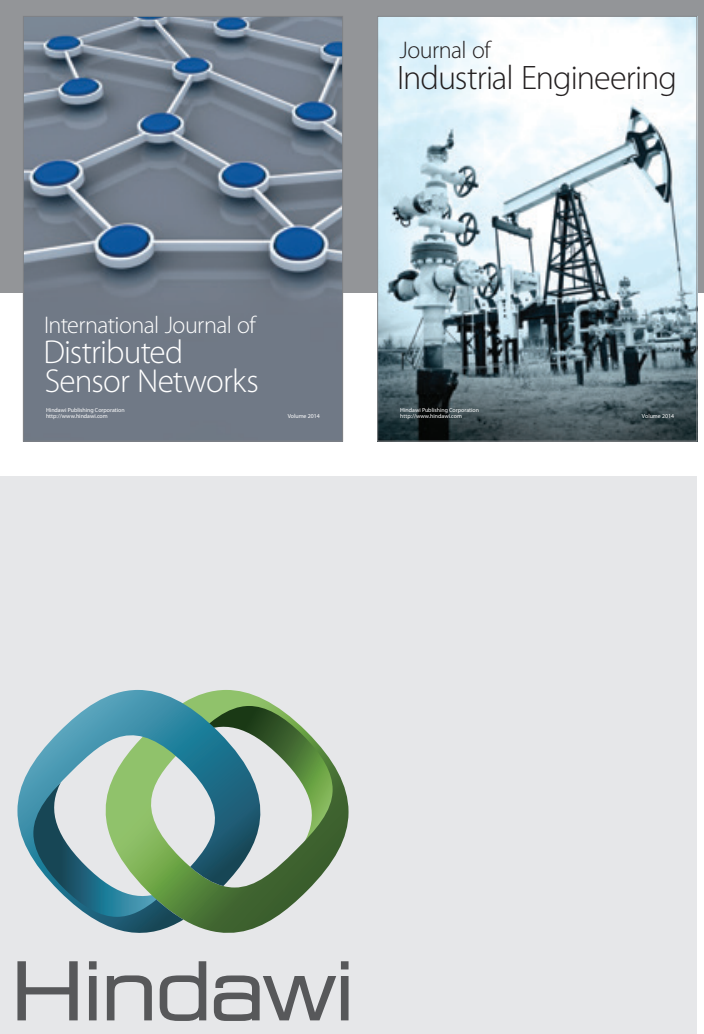

Submit your manuscripts at

http://www.hindawi.com

\section{Computer Networks} and Communications
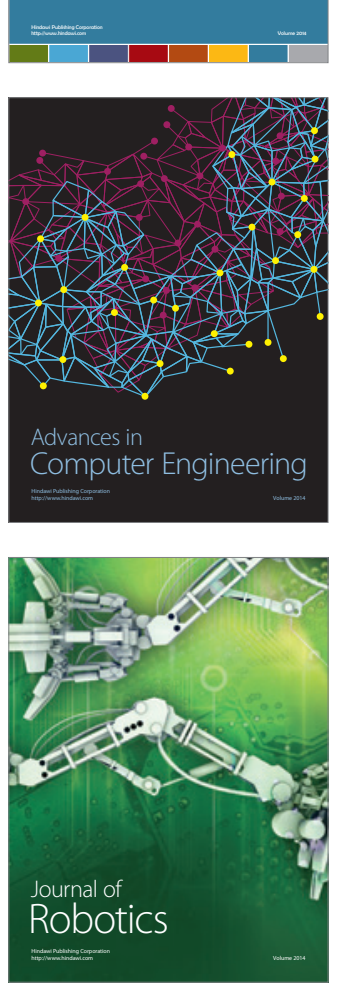
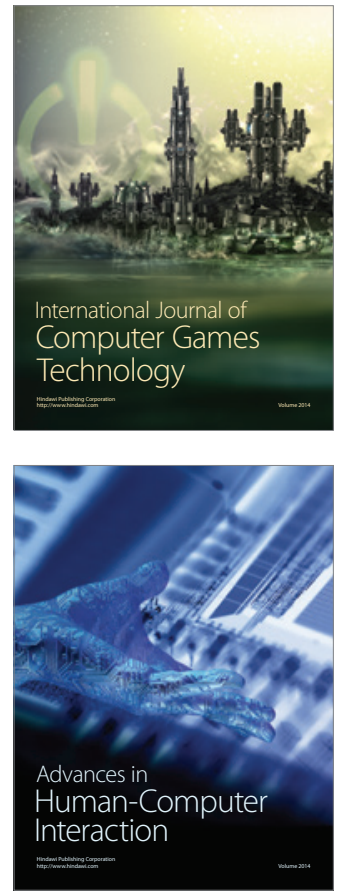
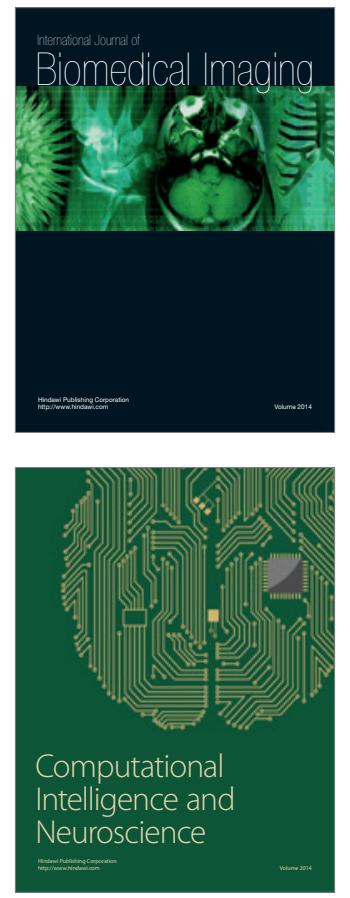
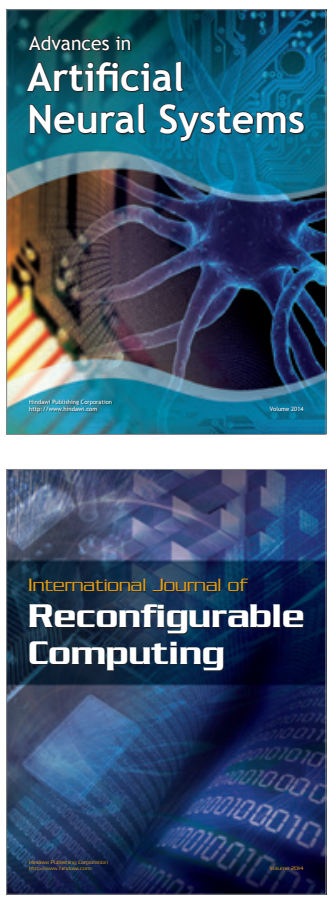
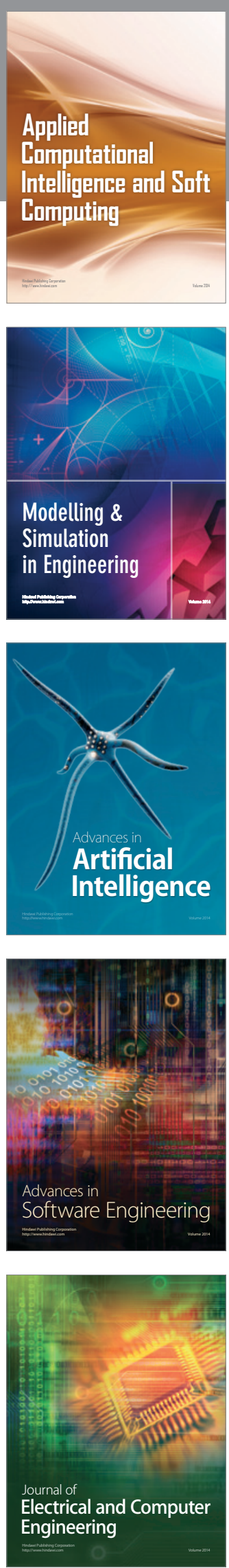